\title{
Erratum: Thermalization of photoexcited carriers in two-dimensional transition metal dichalcogenides and internal quantum efficiency of van der Waals heterostructures [Phys. Rev. Research 2, 043051 (2020)]
}

Dinesh Yadav $\odot$, Maxim Trushin, and Fabian Pauly $\odot$

(Received 15 March 2021; published 25 March 2021; corrected 20 July 2021)

DOI: 10.1103/PhysRevResearch.3.019001

We have found the following mistake in our published paper. The parameters $\lambda_{\mathrm{e}}$ and $\lambda_{\mathrm{h}}$ should be dimensionless, and the corrected Eq. (6) reads (see also Ref. [1], containing the correct definitions)

$$
\begin{array}{ll}
f_{n \mathbf{k}}(0, T)=f_{n \mathbf{k}}^{(0)}(T)-\lambda_{\mathrm{h}} e^{-\frac{\left(\varepsilon_{n \mathbf{k}}+\zeta+\Delta / 2\right)^{2}}{2 \sigma^{2}}}, \quad \varepsilon_{n \mathbf{k}}<\varepsilon_{\mathrm{F}}, \\
f_{n \mathbf{k}}(0, T)=f_{n \mathbf{k}}^{(0)}(T)+\lambda_{\mathrm{e}} e^{-\frac{\left(\varepsilon_{n \mathbf{k}}-\zeta-\Delta / 2\right)^{2}}{2 \sigma^{2}}}, \quad \varepsilon_{n \mathbf{k}} \geqslant \varepsilon_{\mathrm{F}} .
\end{array}
$$

Due to this modification the photoexcited carrier density used in our calculations changes from $7 \times 10^{12} \mathrm{~cm}^{-2}$ to $3.3 \times 10^{14} \mathrm{~cm}^{-2}$ throughout the paper, i.e., it becomes by a factor of $\mathrm{eV} / \sqrt{2 \pi \sigma^{2}} \approx 47$ larger than originally stated. Since this carrier density remains sufficiently low, the relaxation time approximation (RTA) still applies, and neither figures nor outcomes are affected by this change.

[1] D. Yadav, F. Pauly, and M. Trushin, Charge-carrier thermalization in bulk and monolayer CdTe from first principles, Phys. Rev. B 103, 125113 (2021).

Correction: Equation (1) contained two sign errors and has been fixed.

Published by the American Physical Society under the terms of the Creative Commons Attribution 4.0 International license. Further distribution of this work must maintain attribution to the author(s) and the published article's title, journal citation, and DOI. 